\title{
Progress Towards III-V Photovoltaics on Flexible Substrates
}

\author{
Jeremiah S. McNatt ${ }^{1}$, AnnaMaria T. Pal ${ }^{2}$, Eric B. Clark ${ }^{3}$, and Ali Sayir ${ }^{4}$ \\ NASA Glenn Research Center, Cleveland, $\mathrm{OH}, 44135$ \\ Ryne P. Raffaelle ${ }^{5}$, Christopher G. Bailey ${ }^{6}$, and Seth M. Hubbard ${ }^{7}$ \\ Rochester Institute of Technology, Rochester, NY, 14623 \\ William F. Maurer ${ }^{8}$ \\ Ohio Aerospace Institute, Brookpark, OH, 44142 \\ and \\ Les Fritzemeier ${ }^{9}$ \\ Wakonda Technologies, Fairport, NY, 14450
}

\begin{abstract}
Presented here is the recent progress of the NASA Glenn Research Center OMVPE group's efforts in the development of high efficiency thin-film polycrystalline III-V photovoltaics on optimum substrates. By using bulk polycrystalline germanium (Ge) films, devices of high efficiency and low mass will be developed and incorporated onto low-cost flexible substrates. Our progress towards the integration of high efficiency polycrystalline III-V devices and recrystallized Ge films on thin metal foils is discussed.
\end{abstract}

\section{Nomenclature}

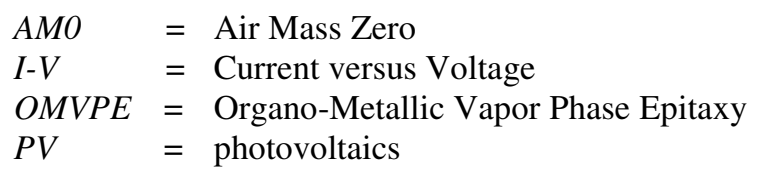

\section{Introduction}

NASA and other government agencies have invested heavily in silicon and II-VI based thin film photovoltaics (PV) technologies with the hopes of delivering both high efficiency $(\sim 15 \%)$ and low mass for both space and terrestrial applications. Despite significant investment, device efficiencies have failed to meet target goals and have demonstrated limited progress in recent years. This has led most agencies to divest themselves of thin film PV research programs. The traditional approaches have tried to make something that is inherently lightweight, highly efficient. Our approach is to take something with demonstrated high efficiency and make it lightweight and flexible.

Electrical Engineer, Photovoltaics and Power Technologies Branch, MS 302-1.

Electrical Engineer, Photovoltaics and Power Technologies Branch, MS 302-1.

Electrical Engineer, Photovoltaics and Power Technologies Branch, MS 302-1.

Ceramic Engineer, Ceramics Branch, MS 106-5.

Professor, Physics Department.

Graduate Student, Microsystems Engineering.

Assistant Professor, Physics Department.

Engineer, Photovoltaics and Power Technology Branch, MS 302-1.

Founder and CEO.

American Institute of Aeronautics and Astronautics 092407 
For near Earth applications, absolute conversion efficiency has become the dominant metric driving solar cell development. As missions move beyond low Earth orbit, specific mass and stowage volume $\left(\mathrm{W} / \mathrm{kg}\right.$ and $\mathrm{W} / \mathrm{m}^{3}$ ) become ever more important. Fig. 1 shows a comparison of cell mass specific powers for III-V multijunction solar cells deposited on various substrates. Current technology is shown by the $29 \%$ AM0 efficiency on 150 micron Ge substrates $(\sim 450 \mathrm{~W} / \mathrm{kg})$. If silicon is substituted for Ge, the specific power nearly doubles despite a predicted drop in overall efficiency. Transitioning to a metal foil provides the potential for an additional increase in specific power, plus the added feature of flexibility. The impact of the high density of molybdenum can be moderated by the possible addition of a polymer (Kapton) backing layer following device fabrications. Finally, if a high temperature polymer substrate is developed, very high mass specific power may be achievable.

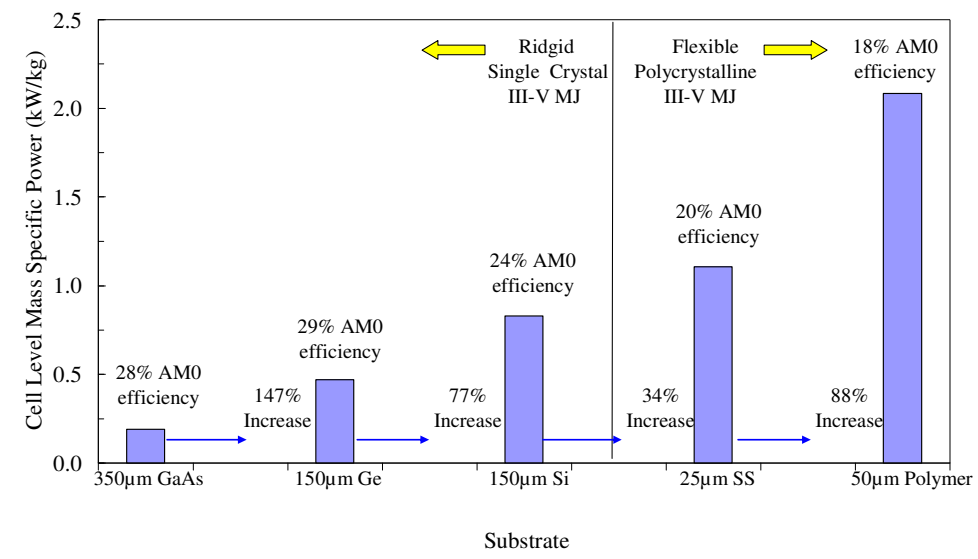

Figure 1. Cell level mass specific power for various thin film substrates.

To reach the high mass specific power metric, we are integrating two demonstrated technologies, (1) recrystallized germanium (Ge) films on thin metal foils and (2) high efficiency polycrystalline III-V photovoltaics, into a high specific power (> $1000 \mathrm{~W} / \mathrm{kg}$ ), high efficiency ( $>20 \%$ AM0), flexible solar array technology. Several research groups have demonstrated the unique ability of thin Ge films deposited on metal or ceramic substrates to form large crystal grains $\left(>1 \mathrm{~mm}^{2}\right)$ under the appropriate thermal annealing conditions $\mathrm{s}^{1-2}$. In addition, we have demonstrated high efficiency III-V photovoltaics (gallium arsenide - GaAs) on bulk polycrystalline Ge wafers with $\sim 1 \mathrm{~mm}^{2}$ average grain sizes. The successful integration of these two technologies appears eminently feasible and highly desirable. The first steps down this path have been taken with the successful demonstration of III-V single and multi-junction devices on Si substrate ${ }^{-3-5}$. Moving to the next step, polycrystalline III-V devices on Mo foil, builds upon component technologies demonstrated previously.

In order to reach the efficiencies projected (20\%), we are developing an indium gallium phosphide (InGaP) device to integrate on top of our existing GaAs device, thus forming a high efficiency multijunction or tandem solar cell. InGaP/GaAs tandem devices have become the industry standard for space photovoltaics. Our previous polycrystalline GaAs devices included InGaP passivating window layers, thus we have confidence that this material can be grown in a polycrystalline format and that a high efficiency device can be developed. At the completion of the effort, a high efficiency (20\% AM0) polycrystalline InGaP/GaAs tandem will be fabricated on the recrystallized Ge film on Mo foil.

\section{Experimental}

Significant progress has been made on the development of recrystallized germanium thin films on molybdenum substrates. Early work included a systematic variation in recrystallization processes (layer structure, layer thicknesses, annealing temperatures and annealing environments), which have better focused our efforts. Our current method follows the structure seen in Fig. 2 where a polished Mo disk has been sputter coated with an ultra-thin W-Ti layer and a thin Ge surface layer. The sample is then annealed at $675{ }^{\circ} \mathrm{C}$ in a $\mathrm{H}_{2} / \mathrm{AsH}_{3}$ environment for 15 minutes. During annealing, As diffuses into the Ge layer converting it from ptype conductivity (as deposited) to n-type (as desired). In addition, a

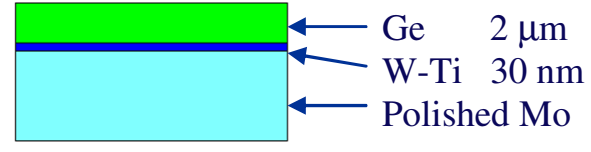

Figure 2. Schematic showing the sample structure and approximate thicknesses of the W-Ti and Ge films.

triggered nucleation of grain growth on the Ge surface is seen. Scanning electron microscopy (SEM) and optical Nomarski microscopy show the diameter of the grains to be greater than $100 \mu \mathrm{m}$ (Fig. 3).

The OMVPE growth of the GaAs based devices followed the conventional practices used in the space photovoltaics industry and can be found elsewhere ${ }^{6}$. The basic cell structure which was used in this work is shown in Fig. 4. Full solar cell devices were grown on both commercial polycrystalline Ge wafers and the previously mentioned recrystallized $\mathrm{Ge}$ on metal foils. The samples were fabricated into working devices following 
conventional photolithography and metallization techniques. These samples were then characterized by standard I-V measurements under a simulated 1 sun AM0 spectrum and by quantum efficiency measurements.

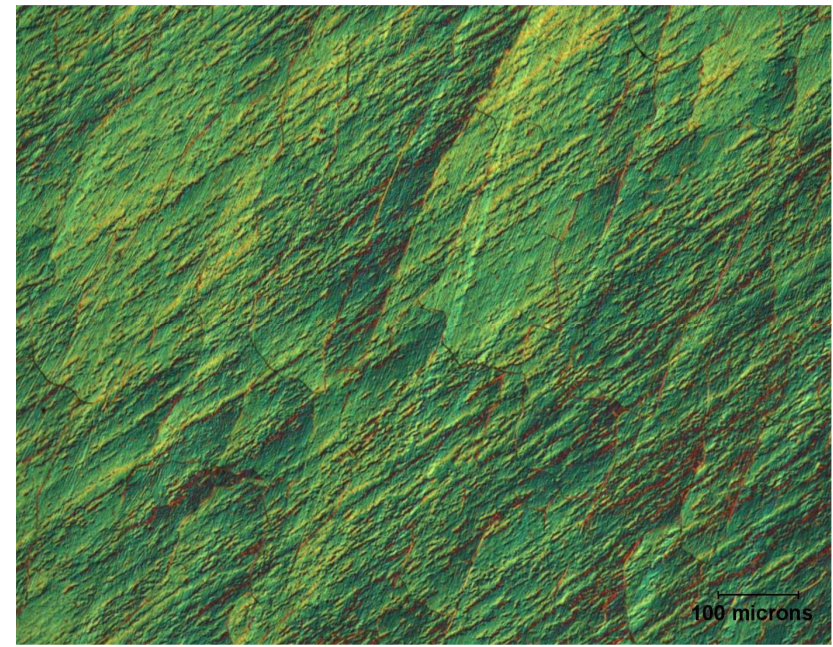

Figure 3. Nomarski microscopy image of the surface of a recrystallized Ge film. The grains are greater than $100 \mathrm{um}$ in diameter.

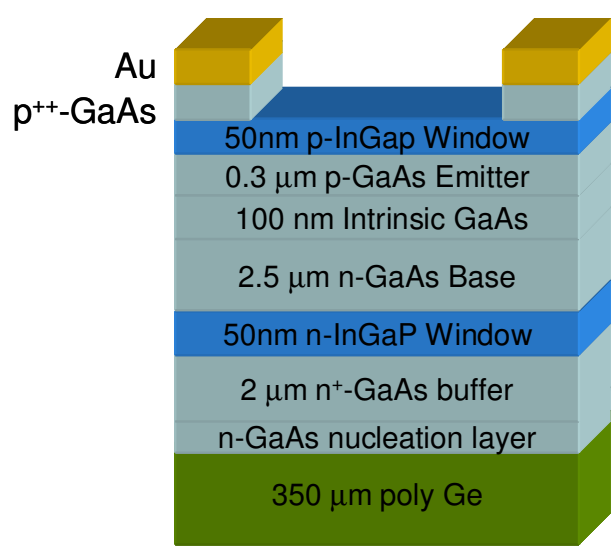

Figure 4. Growth stack schematic for polycrystalline GaAs solar cell

\section{Results}

The efforts to replicate the Ge crystallographic morphology of the commercially available bulk polycrystalline wafers on a thin metal foil are seen in Fig. 5. A nexus of the grain growth is seen at the bottom of the photograph. The annealing did not produce a large degree of grain growth, and the resulting grains were on the order of $10 \mu \mathrm{m}^{2}$ or less. However, the crystallinity of the as-deposited Ge did improve as shown in Fig. 6.

The I-V results of the cell grown on a commercially obtained $350 \mu \mathrm{m}$ thick polycrystalline Ge wafer is shown in Fig. 7. This cell has a very respectable open circuit voltage $\left(\mathrm{V}_{\mathrm{oc}}\right)$ of $\sim 1.0 \mathrm{~V}$ and a short-circuit current density $\left(\mathrm{I}_{\mathrm{sc}}\right)$, without anti-reflective coating of $23 \mathrm{~mA} / \mathrm{cm}_{2}$, under a simulated 1 sun AM0 spectrum. Accounting for reflectance losses, it is estimated that this cell would have approximately a $17 \%$ AM0 conversion efficiency.

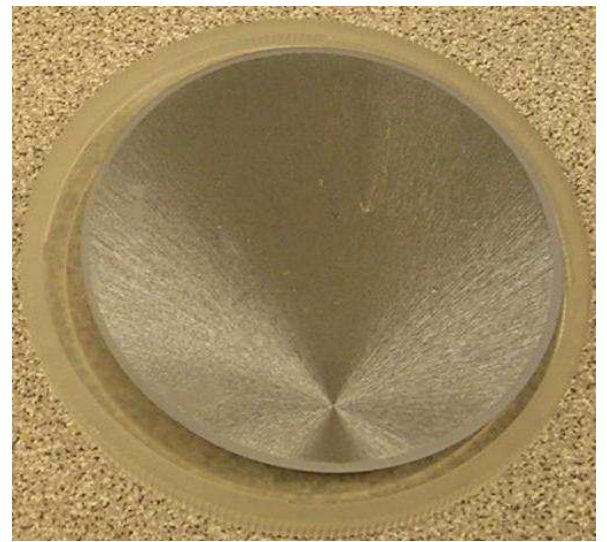

Figure 5. A digital photograph of the Ge/WTi/Mo foil, where a nexus of grain growth is observed in near the edge at the bottom of the image.

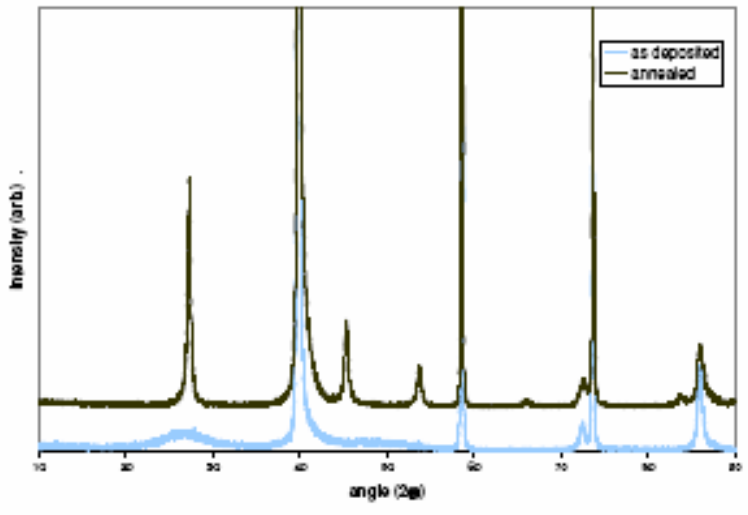

Figure 6. X-ray diffraction results for as-deposited and Ge films anneals at $675^{\circ} \mathrm{C}$ in $\mathrm{AsH}_{3}$.

The current versus voltage results under a simulated 1 sun AM0 spectrum for the recrystallized Ge cell are given in Fig. 8. Unfortunately there was a substantial decrease in the $\mathrm{I}_{\mathrm{sc}}, \mathrm{V}_{\mathrm{oc}}$, and the fill factor over the control 
polycrystalline device. Although the actual device performance was limited, this demonstration a thin film lightweight and flexible III-V device on a low-cost metallic substrate did achieve a specific power comparable to the best space PV devices currently available. Spectral response data showed no surprises and basically had a uniformly reduced intensity over the approximately 370 to $910 \mathrm{~nm}$ range of interest. These initial results were undoubtedly limited by the small grain sizes and rough morphology achieved by this approach. The use of capping layers to promote grain growth and better surface morphology should prove beneficial to improving the performance of this type of a device.

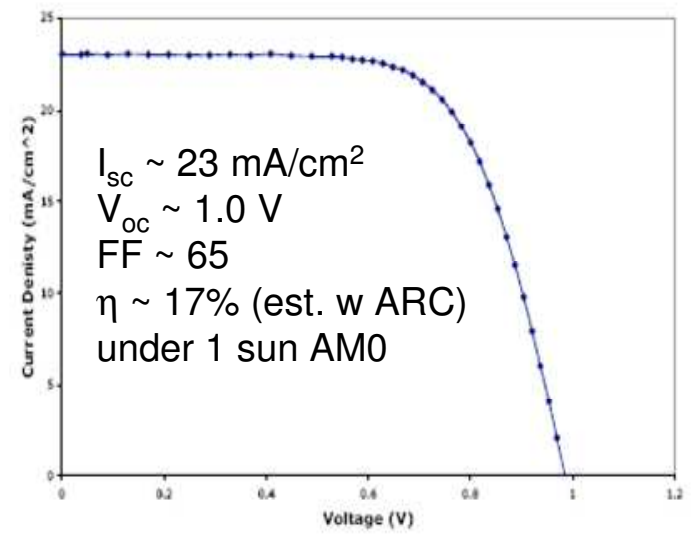

Figure 7. I-V curve for a GaAs cell deposited on a 350 um commercial polycrystalline Ge substrate.

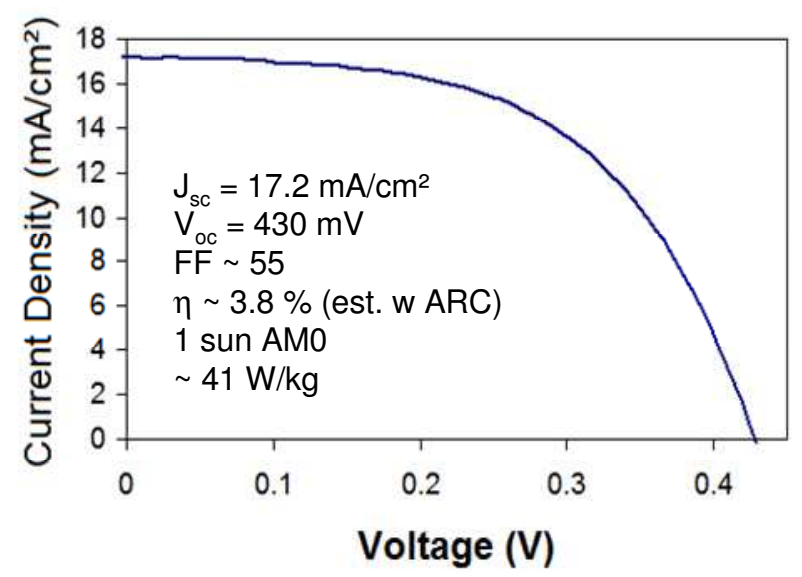

Figure 8. Current versus voltage results for a GaAs p/i/n solar cell grown on a Ge/Ti-W/Mo foil substrate.

Optimization of GaAs cell performance is continuing with the optimization of base doping levels, base dopant species and overall device structure. We are currently in the process of developing a hydrogen sulfide $\left(\mathrm{H}_{2} \mathrm{~S}\right)$ dopant source. It is hoped that sulfur will diffuse to the grain boundaries and create a passivating electric field which will improve the cell performance. We have also recently produced a heterojunction GaAs wherein the GaAs emitter was replaced with a InGaP emitter. This device demonstrated an enhanced Voc ( $80 \mathrm{mV}$ increase) despite having a lower current due to absorption losses in the InGaP emitter. These losses will not negatively impact multijunction devices and thus may be incorporated in future multijunctions.

\section{Conclusion}

A GaAs-based p-i-n solar cell which had a $\mathrm{V}_{\mathrm{oc}}$ of approximately $1.0 \mathrm{~V}$ and $\mathrm{I}_{\mathrm{sc}}$ of nearly $23 \mathrm{~mA} / \mathrm{cm}^{2}$ (device without antireflection (AR) coating) under 1 sun AM0 illumination was produced on a commercial polycrystalline Ge wafer. This result translates to an estimated AM0 efficiency of nearly $17 \%$ using a suitable AR coating. This same device structure was used to produce a solar cell on a re-crystallized Ge thin film on a Mo foil substrate, and yielded a specific power of over $40 \mathrm{~W} / \mathrm{kg}$ when measured under a simulated 1 sun AM0 spectrum. This result, albeit somewhat modest, is nonetheless encouraging when considering the small grain size of the polycrystalline Ge film, and obvious problems seen in surface morphology of the foils used to date.

\section{References}

${ }^{1}$ Ohmachi, Y., Nishioka, T., Shinoda, Y., “Zone-Melting Germanium Film Crystallization with Tungsten Encapsulation,” Appl. Phys. Lett. Vol. 43, No. 10, 15 Nov. 1983 pp. 971-973.

${ }^{2}$ Mauk, M. G., Balliet, J. R., Feyock, B. W., "Large-Grain (>1-mm), Recrystallized Germanium Films on Alumina, Fused Silica, Oxide-Coated Silicon Substrates for III-V Solar Cell Applications,” J. Crystal Growth, Vol. 250, No. 1-2, Mar. 2003, pp. $50-56$.

${ }^{3}$ Wilt, D. M., Pal, A. T., Prokop, N. F., et. al, “Thermal Cycle Testing of GaAs on Si and Metamorphic Tandem on Si Solar 
Cells," Proceedings of the $31^{\text {st }}$ IEEE Photovoltaic Specialists Conference, Jan 2005, pp. 571-574.

${ }^{4}$ Ringel, S. A., Andre, C. L., Fitzgerald, E. A., Pitera, A. J., Wilt, D. M., "Multi-Junction III-V Photovoltaics on LatticeEngineered Si Substrates," Proceedings of the 31 ${ }^{\text {st }}$ IEEE Photovoltaic Specialists Conference, Jan. 2005, pp. 567-570.

${ }^{5}$ Walters, R. J., Garner, J. C., Lam S. N., et. al, "Materials on the International Space Station—Forward Technology Solar Cell Experiment," Materials Science and Engineering B, Vol. 116, No. 3, 15 Feb. 2005, pp. 257-263.

${ }^{6}$ Wilt, D. M., Smith, M. A., Maurer W. F., Scheiman, D. A., Jenkins, P. J., “GaAs Photovoltaics on Polycrystalline Ge Substrates," Conference Record of the 2006 IEEE 4th World Conference, Vol. 2, May 2006, pp. 1891-1894. 